Vol. 2 Issue 1, Jan.2012, pp. 035-037

\title{
Rutishauser method and Gerschgorin circles method to compute eigenvalues of arbitrary matrices
}

\author{
T.D.Roopamala ${ }^{1}$, S.K.Katti ${ }^{2}$ \\ ${ }^{1}$ (Department,of computer science and Engg., Mysore University, INDIA) \\ ${ }^{2}$ (Research supervisor/ Mysore University, INDIA)
}

\begin{abstract}
In this paper a graphical technique using Gerschgorin circles to computed eigenvalues of a system matrix is presented. The results have been compared with the existing method. A suitable step length has been taken at the Gerschgorin bound and in this step length the Bisection method is applied to compute the eigenvalues of the system matrix.
\end{abstract}

Keywords - Gerschgorin circles, system matrix, eigenvalues, Gerschgorin bound, Bisection method

\section{INTRODUCTION}

. In literature there exist several methods to compute the eigenvalues of the system matrix [2,3]. In Rutishauser method [1] the given matrix are transformed until the lower and upper triangular elements are less 0.05 in magnitude. In this approach the elements in the lower and upper triangular are almost tending to zero but not exactly to zero. Hence the eigenvalues obtained will not be very accurate, but closer to accuracy. In control system and computer application eigenvalues play very important role. If the eigenvalues are closer to the imaginary axis exact eigenvalues has to be considered to decide the stability of the system. In this paper , the existing method available in [1] has been explained by taking a example and the proposed method has been applied to the same example and the results are compared.

\section{EXISTING METHOD}

Consider a system matrix $\mathrm{A}$ of order $(3 \times 3)$

$$
A=\left(\begin{array}{lll}
1 & 1 & 1 \\
2 & 1 & 2 \\
1 & 3 & 2
\end{array}\right)
$$

Using the Rutishauser method .Iterate till the elements in the lower triangular part are less than 0.05 in magnitude.

We have

$$
A_{1}=A=\left(\begin{array}{ccc}
1 & 0 & 0 \\
2 & 1 & 0 \\
1 & -2 & 1
\end{array}\right) \quad\left(\begin{array}{ccc}
1 & 1 & 1 \\
0 & -1 & 0 \\
0 & 0 & 1
\end{array}\right)
$$
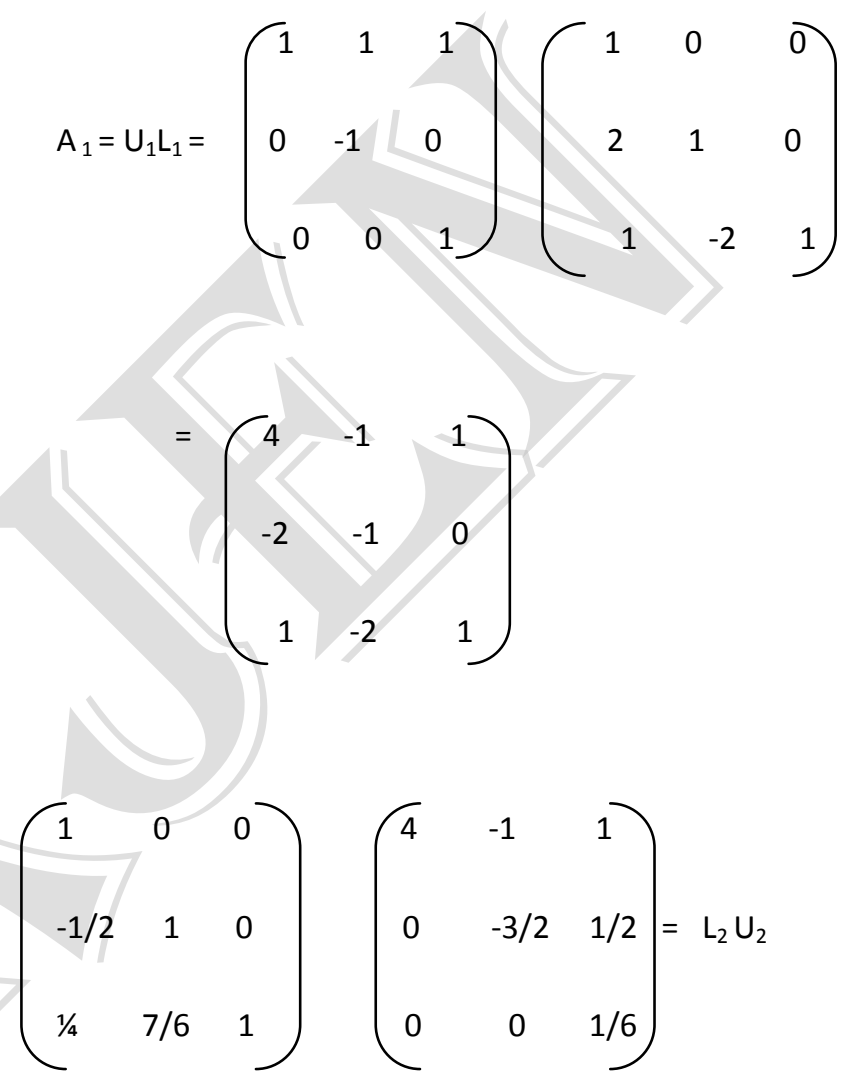
T.D.Roopamala, S.K.Katti / IOSR Journal of Engineering (IOSRJEN)

www.iosrjen.org

ISSN : 2250-3021

Vol. 2 Issue 1, Jan.2012, pp. 035-037

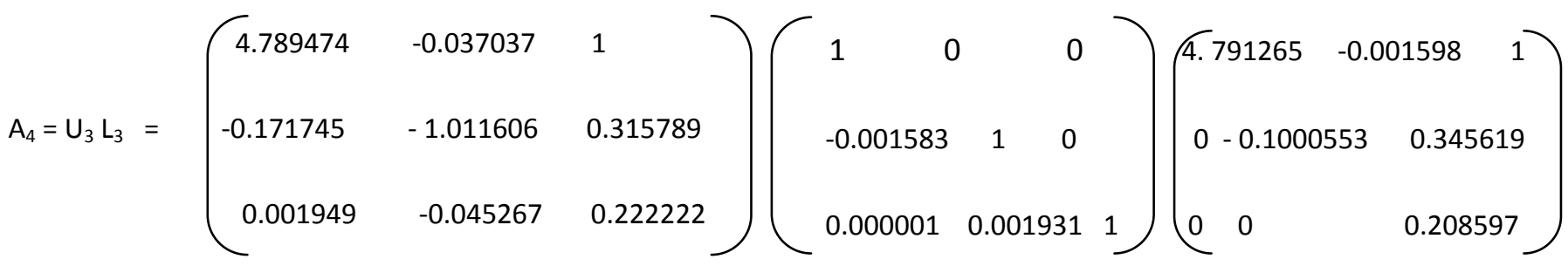

$\left(\begin{array}{ccc}1 & 0 & 0 \\ 0.035859 & 1 & 0 \\ 0.000407 & 0.044670 & 1\end{array}\right)\left(\begin{array}{cccc}4.789474 & -0.037037 & 1 \\ 0 & --1.11606 & 0.351648 \\ 0 & 0 & 0.206107\end{array}\right) A_{7}=U_{6} L_{6}=\left(\begin{array}{cccc}4.791289 & 0.000333 & 1 \\ 0.001584 & -0.999886 & 0.345619 \\ 0.000084 & 0.000403 & 0.208597\end{array}\right)$

$=\mathrm{L}_{4} \mathrm{U}_{4}$

Hence the eigenvalues of A are approximately 4.791289 , 0.999886 and 0.208579

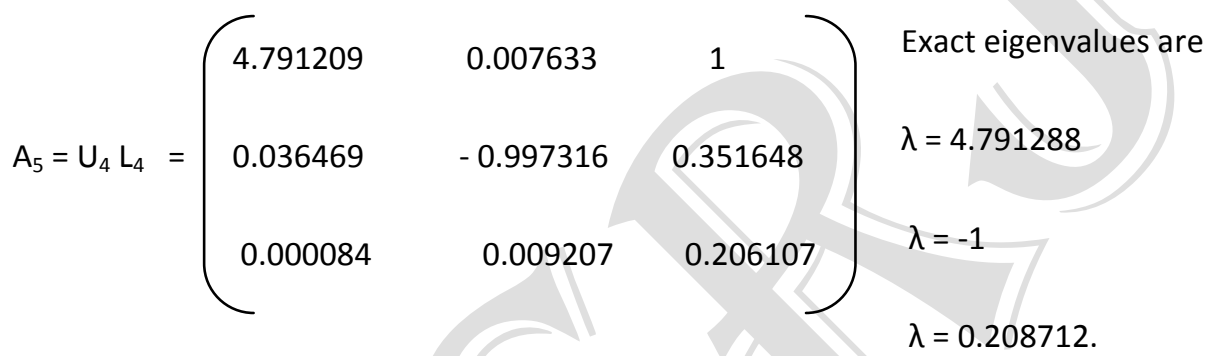

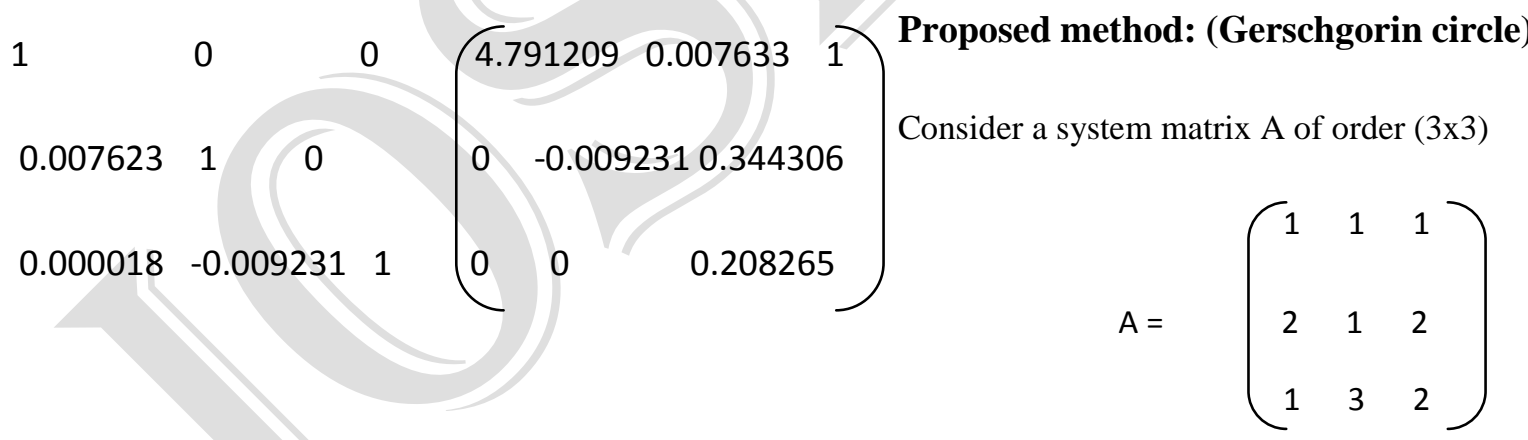

$=\mathrm{L}_{5} \mathrm{U}_{5}$

$A_{6}=U_{5} L_{5}=\left(\begin{array}{ccc}4.791209 & 0.007633 & 1 \\ 0.036469 & -0.997316 & 0.351648 \\ 0.000084 & 0.009207 & 0.206107\end{array}\right)$

Gerschgorin circles of the above matrix 
Vol. 2 Issue 1, Jan.2012, pp. 035-037

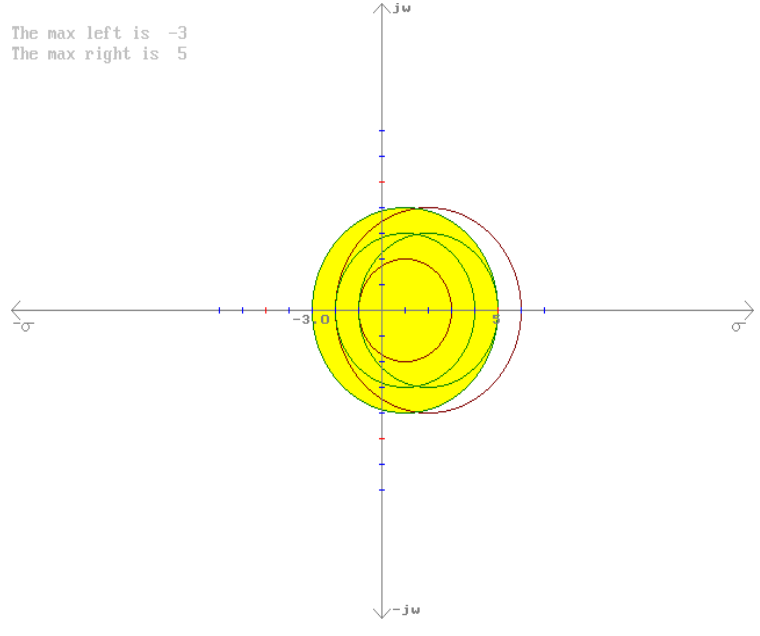

Gerschgorin bound $[-3,5]$

Eigenvalues of the above by applying step length $\mathrm{h}=1.0$ and Bisection method are

$\lambda=4.791992$

$\lambda=-1$

$\lambda=0.208008$.

Total computation: 339

Conclusion: We can observe that the existing method converts the given matrix as the product of lower and upper triangular matrix for seven times which is a tedious process to compute the eigenvalues and the values are closer to the exact value.

In the proposed method the computation of eigenvalues is very simple and the Bisection technique is applied at the Gerschgorin bounds with the step length one. The eigenvalues compared with the exact values are very closer to the exact and one of the eigenvalues is exactly same. Hence Gerschgorin technique is simpler and calculates the eigenvalues accurately compared to the existing method.

\section{References}

[1] M. K. Jain, S. R. K. Iyengar and R. K. Jain, "Numerical Methods for Scientific and Engineering Computation", Wiley Eastern Limited, 1993.pp.201-203

[2] X. H. Sun et al., "Efficient tridiagonal solvers on multicomputer", IEEE Transactions on Computers, Vol. 41, no. 3, 1992, pp. 286-296.

[3] Y. Juang and W. F. McColl," A two-way BSP algorithm for tridiagonal systems", Future Generation Computer Systems, Vol.13, Issues 4-5, 1998, pp. 337347

[4] V. S. Pusadkar and S. K. Katti, "A New Computational Technique to identify real eigenvalues of the system matrix A", via Gerschgorin theorem, Journal of
Institution of Engineers (India), Vol. 78, Dec. 1997, pp. 121-123.

[5] Y.V. Hote,"New Approach of Kharitonov and Gerschgorin theorem in Control Systems", Ph. D thesis, Delhi University, 2009.

[6] Y. V. Hote D. Roy Choudhury, and J. R. P. Gupta, "Gerschgorin theorem and its applications in control system problems", IEEE International Conference on Industrial Technology, 2006, pp. 2438-2443.

[7] T.D.Roopamala and S.K.Katti, "Eigenvalues of tridiagonal matrix using Strum sequences and Gerschgorin Theorem" IJCSE, Vol3.No.12.Dec 2011 .pp.3722-3727.

[8] T.D.Roopamala and S.K.Katti, "Comments on "Computation of range of interval between in which the roots lie by Given's technique “, IJCSNS ,Vol.10, No.3.pp.206-.207 March 2010. 